\title{
Resilience as a mediator between extraversion and life satisfaction among Chinese college students
}

\author{
Jing Jie ${ }^{1, a}$, Jie $\mathrm{Du}^{2, \mathrm{~b},{ }^{*}}$ \\ ${ }^{1}$ Mental Health Education Center for College Students, Department of Student Affairs, Hainan \\ University, Haikou, 570228, China \\ ${ }^{2}$ College of Materials and Chemistry Engineering, Hainan University, Haikou, 570228, China

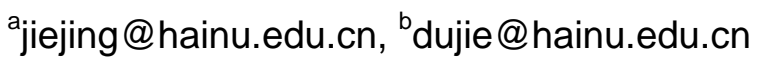

Keywords: resilience, extraversion, life satisfaction, personality, college students

\begin{abstract}
Extraversion was proved to be one of the best predictor of life satisfaction. But this predictive effect could be affected by resilience. In order to examine the relation between the extraversion and life satisfaction, and test the mediate effect of resilience, we chose 110 undergraduate students from Hainan University to complete a battery of tests, including the Extraversion subscale of NEO-Five-Factor-Inventory, the Resilience Scale for Chinese Adolescents and the Life satisfaction Scale for Chinese Adolescents. The results showed that: (1) Extraversion was significantly positive correlated with life satisfaction and resilience.(2) Resilience was significantly positive correlated with general life satisfaction.(3) Regression analyses results revealed that resilience mediated the relation between Extraversion and life satisfaction.
\end{abstract}

\section{Introduction}

The development of positive psychology has provoked a growing interest in the studies of what constitutes people's happiness and well-being [1]. Life satisfaction is an essential element of positive well-being. Recently, it is of considerable interest to investigate the relationship of SWB with personality [2]. According to Five Factor Model of personality, individual differences in personality may be described along the factors of conscientiousness, agreeableness, neuroticism, openness to experience, and extraversion [3]. From this point of view, many studies showed that personality is one of the foremost predictors of SWB, especially extraversion.

Extraversion is a kind of personality trait including the quantity and intensity of relationships (such as sociability and dominance), energy level, positive emotionality, and excitement seeking [2]. As a higher level of happiness and positive affect than introverts, extraversion is also assumed to be correlated with life satisfaction. Children with more relaxed, extraverted dispositions showed higher life satisfaction [4]. An early study by Costa and McCrae also demonstrated relationships among extraversion and life satisfaction [5]. Subsequently, numerous studies have replicated this result.

However, some evidence showed that personality is related to resilience. Resilience has been defined as resistance to illness, adaptation, and thriving, the ability to bounce back from adversity or recover from stress is closest to its original meaning[6]. Fredrickson, et al. found that resilient students' levels of life satisfaction were significantly higher than those of low-resilient students [7]. King concluded that not only was resiliency positively related to life satisfaction among individuals experiencing divorce, but also resiliency significantly predicted life satisfaction [8]. Resilience was also found to be linked to extraversion[9].

Thus extraversion and resilience both have relationship with his/her life satisfaction. Although this viewpoint has been identified by many researchers, there are some academic queries still alive. For instance, which factor plays a decisive role for life satisfaction? Does extraversion have an indirect influence on life satisfaction? Is the effect of extraversion on life satisfaction actually mediated by resilience? To deal with these queries, we explore the relationship between extraversion, resilience and life satisfaction for college students. To our knowledge, this is the first study to explore the mediate role of resilience in the relationship between extraversion and life satisfaction. 
Our hypothesis is that the relationship between extraversion and life satisfaction could be explained by resilience. More specifically, although the initial correlation between extraversion and life satisfaction is expected to be significant, it is anticipated to drop to a non-significant level once resilience is entered into the regression equation as a mediator. This means that the direct effects model is incomplete, because the effect of extraversion on life satisfaction is actually mediated by resilience.

\section{Method}

\subsection{Participants}

A total of 110 Hainan University undergraduate students completed a battery of questionnaires. The sample consisted of 44 males(40\%), 66 females(60\%), ranging in age from 18 to 23 years $(M=20.72$, $\mathrm{SD}=0.826)$.

\subsection{Measures}

2.2.1 The Extraversion Subscale of NEO-Five-Factor-Inventory(Costa and McCrae's, 1992)

Extraversion was assessed by the Chinese translation of Costa and McCrae's (1992) extraversion subscale of NEO-Five-Factor-Inventory. It contains 12 items. The Simplified Chinese Version of NEO-FFI was offered by Psychological Assessment Resource, Inc.

2.2.2 Resilience Scale for Chinese Adolescents(Hu Y Q and Gan Y Q,2008)[10]

The 27 items of the RS(Chinese Version)are organized into five factors(goal planning, help-seeking, family support, affect control, and positive thinking), asking respondents to rate themselves along 27 statements on a 5-point Likert scale $(1=$ totally incorrect, $5=$ totally correct $)$

2.2.3. Life satisfaction Scale for Chinese Adolescents(Zhang X G et al.,2004)[27]

Life satisfaction was measured by the Life satisfaction Scale for Chinese Adolescents. The scale asks respondents to rate themselves along 36 statements on a 7 -point Likert scale $(1=$ totally incorrect,7= totally correct).

2.3 Procedure

Participants received a questionnaire directly after regular classes. All respondents were informed that participation was voluntary and anonymous and told approximately how much time it would take to complete the questionnaire. All participants completed the questionnaires in a quiet environment with a researcher present. It took about 20 minutes for the participants to fill out the questionnaire.

2.4 Data analysis

All the data were analyzed using SPSS version 18.0.Descriptive analyses correlation analyses were performed on all variables. Logistic regression analyses were performed to find out the effects of resilience on conscientiousness and life satisfaction, controlling for gender, age and so on. The odds ratios and corresponding 95\% confidence intervals were calculated. $\mathrm{P}<0.05$ was considered significant for all tests.

\section{Results}

\subsection{Correlations}

Means and standard deviations, internal consistencies, and intercorrelations are listed in Table 1.

Extraversion correlated strongly with both resilience and life satisfaction $(r=0.293, p<0.01$ and $r$ $=0.259, \mathrm{p}<0.01)$. The correlation between resilience and life satisfaction was significant $(\mathrm{r}=0.651, \mathrm{p}$ $<0.001)$.

Table 1. Descriptive statistics and correlations between measures $(\mathrm{N}=110)$

\begin{tabular}{cccccc}
\hline & M & SD & Extraversion & Resilience & Life Satisfaction \\
\hline Extraversion & 37.496 & 3.910 & 1 & $0.293^{* * * *}$ & $0.259^{* *}$ \\
Resilience & 98.760 & 10.572 & & 1 & $0.651^{* * *}$ \\
Life Satisfaction & 171.420 & 19.301 & & & 1 \\
\hline
\end{tabular}

Note:* $\mathrm{p}<.05 ; * * \mathrm{p}<.01 ; * * \mathrm{p}<.001$. 


\subsection{Regression Analyses}

The power of life satisfaction predictors might change with demographic characteristics such as gender, age and so on. Therefore, the present investigation controls the influence of these variables by statistical procedures. The result of the hierarchical regression on life satisfaction is shown in Table 2 .

TABLE 2 Summary of hierarchical regression analysis for predicting life satisfaction $(\mathrm{N}=110)$

\begin{tabular}{|c|c|c|c|c|c|c|}
\hline $\begin{array}{l}\text { dependent } \\
\text { variable }\end{array}$ & $\begin{array}{l}\text { independent } \\
\text { Variable }\end{array}$ & $B$ & $S E B$ & $\beta$ & $t$ & $\begin{array}{l}\text { Total } \\
R 2\end{array}$ \\
\hline Step 1 & & & & & & \\
\hline $\begin{array}{l}\text { Life Satisfaction } \\
\text { Step } 2\end{array}$ & Extraversion & 1.386 & 0.466 & 0.279 & $2.971 * *$ & 0.093 \\
\hline $\begin{array}{l}\text { Resilience } \\
\text { Step } 3\end{array}$ & Extraversion & 0.802 & 0.255 & 0.295 & $3.148 * *$ & 0.096 \\
\hline $\begin{array}{l}\text { Life Satisfaction } \\
\text { Step } 4\end{array}$ & Resilience & 1.195 & 0.135 & 0.654 & $8.863 * * *$ & 0.440 \\
\hline Life Satisfaction & $\begin{array}{l}\text { Extraversion } \\
\text { and Resilience }\end{array}$ & $\begin{array}{l}0.468 \\
1.144\end{array}$ & $\begin{array}{l}0.383 \\
0.141\end{array}$ & $\begin{array}{l}0.094 \\
0.626\end{array}$ & $\begin{array}{l}1.222 \\
8.128 * * *\end{array}$ & 0.448 \\
\hline
\end{tabular}

Note:* $\mathrm{p}<.05 ; * * \mathrm{p}<.01 ; * * * \mathrm{p}<.001$.

The first set of equations regressed extraversion variable on life satisfaction. Extraversion accounted for a significant amount of variance $(9.3 \%)$ in life satisfaction.

The second set of equations regressed extraversion on resilience showed a significant portion of the variance $(9.6 \%)$.

The third series equation regressed resilience on life satisfaction revealed a significant portion of the variance $(44 \%)$ in life satisfaction.

The fourth set of equation regressed on extraversion and resilience of life satisfaction. The model was significant and accounted for $44.8 \%$ of the variance in life satisfaction. Then we compared the beta weights in the model (i.e. beta weights for extraversion alone on life satisfaction and extraversion when resilience were also included in the equation). The beta weight of extraversion was regressed alone on life satisfaction was 0.279 . When resilience was included in the equation, the resulting beta weight was 0.094, suggesting that resilience acts as a mediator in the relationship between extraversion and life satisfaction. The mediate effect accounted for $66.20 \%$ of the total effect between extraversion and life satisfaction. Figure 1 depicts the test of the mediating effects of resilience in the relationship between extraversion and life satisfaction.
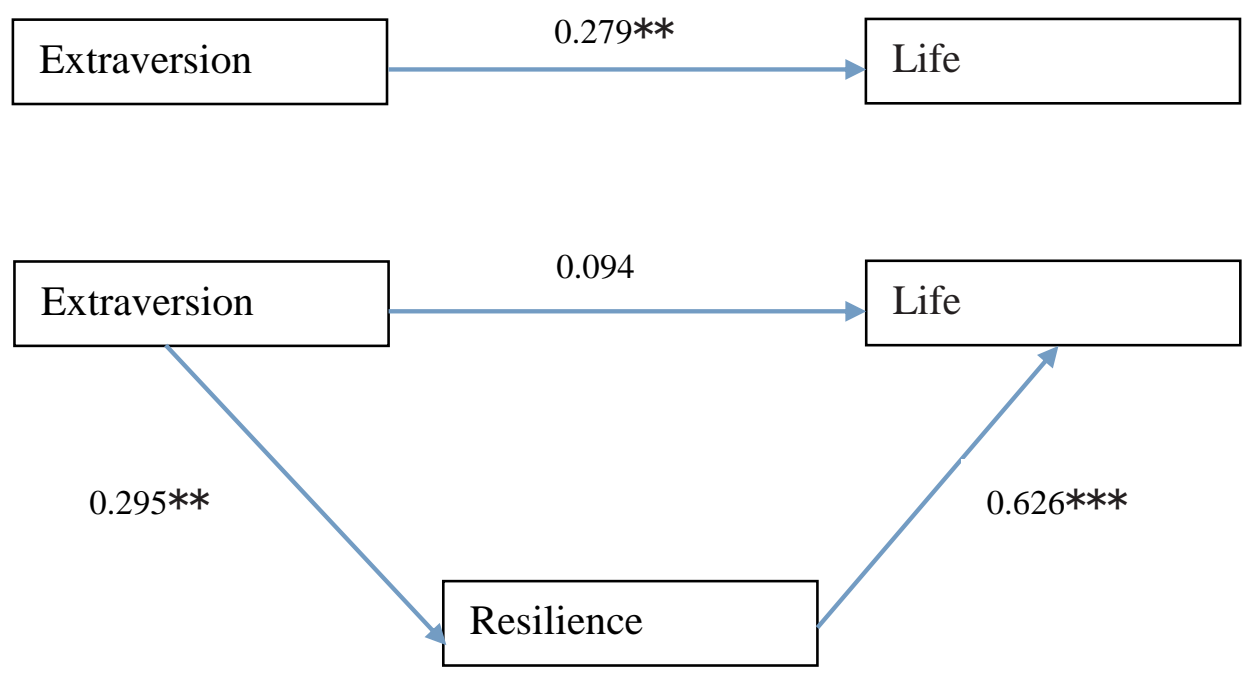

Figure 1. Path model of the mediational role of resilience in the relation between extrversion and life satisfaction. (Note:* $\mathrm{p}<0.05$;** $\mathrm{p}<0.01 ; * * * \mathrm{p}<0.001$. 


\section{Discussion}

The main objective of our study has been to investigate resilience as a mediator of the influence of extraversion on life satisfaction.

On a correlational level, the results revealed significantly positive associations between extraversion, and life satisfaction. Numerous studies have reported that extraversion is positively related to life satisfaction, with little or no contravening evidence $[4,6]$ This finding is also consistent across cultures[11].

Meanwhile we also found extraversion significantly positive correlated with resilience, similar to other results. And the correlation between resilience and life satisfaction was significantly positive. So we hypothesized that the relation between extraversion and life satisfaction could be explained by resilience. Further results of regression analysis confirmed our hypothesis.

The college students scored better resilience, contributing to higher levels of life satisfaction. Moreover, resilient people were found to possess a host of psychological resources, including optimism, tranquility, low neuroticism and high openness. They are more likely to find positive meaning in problems they have to face [8]. These attributes of resilient people were contributed to higher levels of life satisfaction.

Resilience was never treated as the medium variable between the personality and life satisfaction in the past.Some other reports used the top down approaches to emphasize the role of dispositions or traits in individual differences produced in life satisfaction and other well-being variables [1].Differently, we found people with strong extraversion are not only more satisfied with their life, but also higher in resilience which increases their life satisfaction. It means that resilience acts as a mediator between personality and life satisfaction. The results suggest that there is a direct relationship between resilience and life satisfaction, while extraversion and life satisfaction are indirect.

The results implied that besides reducing perceived stress, intervention strategies could also focus on cultivation of resilience. The development of resilience is of great value not only to mitigating maladaptive coping and stress, but also to promoting coping mechanisms [12], resulting in higher levels of life satisfaction.

It means that improving resilience lever could increase the life satisfaction degree. The level of resilience can be enhanced through resiliency training program. For example, a 4-week resilience education program for college students showed significantly higher resilience scores and more effective coping strategies. Employees at a large medical center increased their resilience over a 12-week period by taking part in a self-directed resiliency training program[13].In addition, adolescents significantly enhanced their resilience through various resilience intervention programs[14,15].

Resiliency training program can also improve undergraduate students' life satisfaction, thus affecting their academic performance and adaptation to university life.

There are still several limitations during this study. Firstly, although resilience appears to play an important role for life satisfaction, we cannot conclude, on the basis of our results, that resilience is the only important variable for life satisfaction. In the current study, resilience was associated with $44 \%$ of the variance for life satisfaction. Resilience cannot be taken as a full explanation of life satisfaction. The findings from the current study should be confirmed by prospective cohort studies in the future. And further study should consider other variation among the effect sizes. Secondly, all data were obtained through self reported questionnaires, which could introduce response bias. The participants might have underestimated or over estimated the relationship between the study variables. Thirdly, as the sample was only taken from the Hainan Province in southern China, so the results could not be generalized to the people from other areas or with other cultures. Diener(1996) suggested that personality may predict within-group differences strongly because of the shared environment of that group[16]. So the differences between groups, nations, or cultures(that do not share the same environment), environmental effects are negligible. Some further study are in progress and will be published soon. 


\section{References}

[1]E. Diener,Subjective well-being,Psychological Bulletin.95,(1984) ,542-575.

[2]K. M. DeNeve, H. Cooper,The happy personality: A meta-analysis of 137 personality traits and subjective well-being,Psychological Bulletin. 124,(1998),197-229.

[3]R.R. McCrae, P.T. Jr Costa,Personality trait structure as a human universal, Am Psychol. 52, (1997), 509-516.

[4]E.S.Huebner,Correlates of life satisfaction in children, School Psychology .6,(1991),103-111.

[5]P.Costa,R,McCrae.Influence of extraversion and neuroticism on subjective well-being: Happy and unhappy people, Journal of Personality and Social Psychology .38, (1980),668-678.

[6]M.M. Fredrickson B.L.Tugade, L F Barrett,Psychological resilience and positive emotional granularity:examining the benefits of positive emotions on coping and health, $\mathrm{J}$ Pers. 72, (2004) ,1161-1190.

[7]B. L. Fredrickson, M. M.Tugade, C. E.Waugh , G. R. Larkin, What good are positive emotions in crisis? A prospective study of resilience and emotions following the terrorist attacks on the United States on September 11th,2001, Journal of Personality and Social Psychology.84, 2003), 365-376.

[8] Information on http://commons.lib.niu.edu/handle/10843/10908

[9] L. Riolli, V. Savicki, A. Cepani,Resilience in the face of catastrophe:optimism, personality and coping in the Kosovo crisis,J Appl Soc Psychol. 32,(2002),1604-1627.

[10] Y.Q.Hu, Y.Q.Gan,Development and Psychometric validity of the Resilience Scale for Chinese Adolescent,Acta Psychologica Sinica. 40,(2008),902-912.

[11]U. Schimmack, P.Radhakrishnan, S.Oishi, V. Dzokoto, S. Ahadi, Culture, personality, and subjective well-being: Integrating process models of life satisfaction,Journal of Personality and Social Psychology. 82, (2002), 582-593.

[12]G. Wu, A. Feder, H. Cohen, J.J. Kim, S. Calderon, D.S. Charney, et al. Understanding resilience, Front Behav Neurosci. 7, (2013),10.

[13]V. Sharma, A. Sood, K. Prasad, L. Loehrer, D. Schroeder, B. Brent. Bibliotherapy to decrease stress and anxiety and increase resilience and mindfulness: a pilot trial, Explore (NY). $10,(2014), 248-252$.

[14]R.K. Hodder, J. Daly, M. Freund, J. Bowman, T. Hazell, J. Wiggers, A school-based resilience intervention to decrease tobacco, alcohol and marijuana use in high school students, BMC Public Health. 11,(2011),722.

[15]S.D. Ritchie, M.J. Wabano, K. Russell, L. Enosse, N.L. Young. Promoting resilience and well-being through an outdoor intervention designed for Aboriginal adolescents, Rural Remote Health. 14,(2014),2523.

[16]E. Diener, C. Diener, Most people are happy,Psychological Science. 7, (1996),181- 185. 